\title{
Comparison of Straight and Helical Nanotube Production in a Swirled Fluid CVD Reactor
}

\author{
Graham Bathgate, Sunny Iyuke, and Frank Kavishe \\ Department of Chemical and Metallurgical Engineering, University of the Witwatersrand, 1 Jan Smuts Avenue, Braamfontein, \\ Johannesburg 2000, South Africa
}

Correspondence should be addressed to Sunny Iyuke, sunny.iyuke@wits.ac.za

Received 25 October 2011; Accepted 5 December 2011

Academic Editors: C. A. Charitidis and Y. Yang

Copyright ( $\odot 2012$ Graham Bathgate et al. This is an open access article distributed under the Creative Commons Attribution License, which permits unrestricted use, distribution, and reproduction in any medium, provided the original work is properly cited.

\begin{abstract}
Research into Carbon Nanotubes and their applications is fast becoming an extremely popular topic, and any means to greatly improve the synthesis process has a huge marketability. While investigating the feasibility of continuous production of singlewalled carbon nanotubes in a vertical Swirled Fluid Chemical Vapour Deposition (CVD) reactor, it was discovered that helical nanotubes were lifted from the reactor by the gas current while straight tubes remained behind. Investigation into the merits provided by the helical structure illustrated the greatly increased likeliness for helical tubes to be lifted from the reactor by the carrier gas giving rise to positive speculation of their possible use in vertical CVD reactors in the future.
\end{abstract}

\section{Introduction}

CNTs are allotropes of carbon with a nanostructure that can have a length-to-diameter ratio as large as $28,000,000: 1$, which is unequalled by any other material [1]. The small dimensions, strength, and the remarkable physical properties of these structures allow for a very unique material with a whole range of promising applications [2]. The discovery of single-walled carbon nanotubes (SWCNTs) proved to be an extremely important development since the structures appeared to be approximate to those of the "ideal" nanotubes [3] consisting of tubes with walls only one atom thick. To use Paradise and Goswami's [4] description: "Ideal Nanotubes can be described as a seamless cylinder of rolled up hexagonal networks of carbon atoms." They also describe possible applications ranging from semiconductors, electronic memory, drive products, and medical delivery systems to uses in plastics such as automobile body panels, paint, tires, and as flame retardants in polyethylene and polypropylene.

As SWCNTs are of such importance, there is much marketability for any means by which to improve their synthesis. The best means by which to do this is by developing a fully continuous process. While this process could provide a means to produce larger quantities of SWCNTs, separation and purification is still expensive and so any advantage that results in a purer sample is very desirable. In a vertical reactor, very little is known about the factors that affect which nanotubes will be lifted from the reactor which gives rise to the possibility that given more knowledge, control of certain factors such as shape may provide the means to produce purer products.

\section{Process Overview}

SWCNTs are a very important variety of carbon nanotube because they possess important electronic properties that the multiwalled carbon nanotube (MWCNT) variants do not possess. Single-walled nanotubes are the most likely candidate for miniaturizing electronics beyond the microelectromechanical scale that is currently the basis of modern electronics [5]. Since SWCNTs can be excellent conductors, they can be of great use in miniaturizing the most basic component of these systems, namely, the electric wire. A useful application of SWCNTs is in the development of the first intramolecular field effect transistors (FETs). The production of the first intramolecular logic gate using SWCNT FETs has recently become possible as well [6]. These discoveries can also be of great use in other related areas 


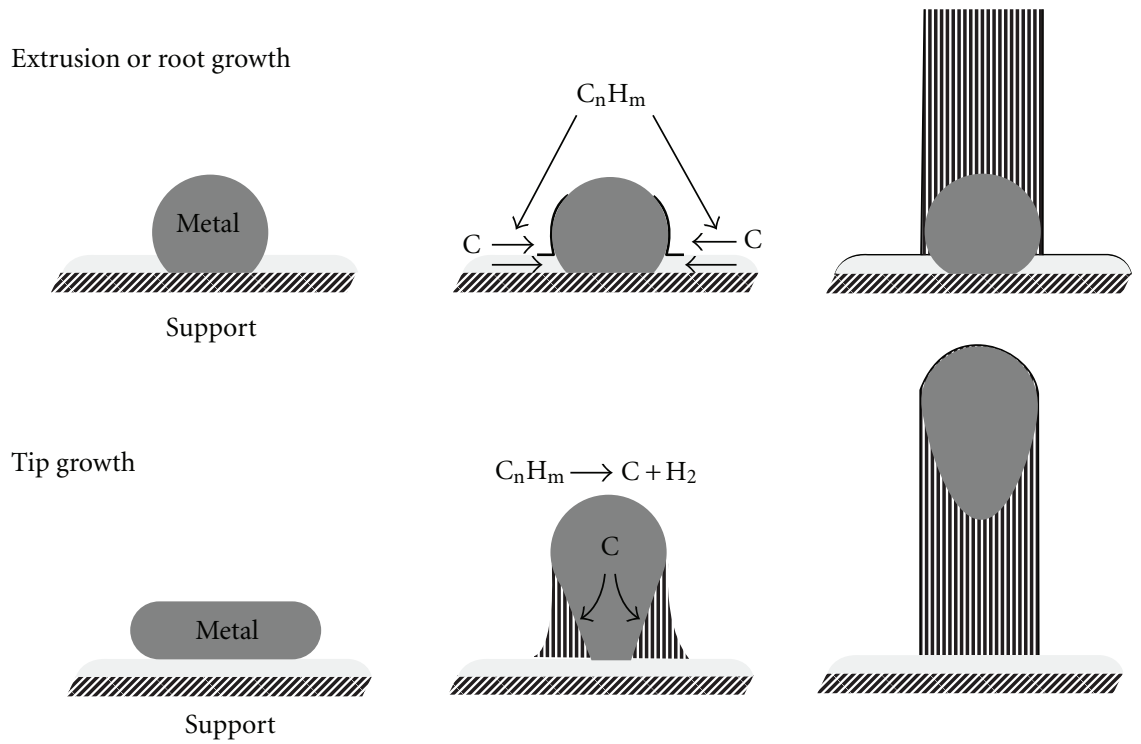

FIgURE 1: Growth mechanisms of carbon nanotubes.

such as for the improvement and minimization of biometric security systems.

In order to optimize the production of carbon nanotubes, the mechanisms by which they are grown need to be fully understood [7]. One mechanism based on the processes involved in carbon fiber formation is described by Sinnott et al. [8] and carbon nanotubes formation described by Zhao et al. [9] whereby carbon diffuses into nanometer-scale catalytic particles. The catalyst particles then realign the carbon atoms on a certain catalytic crystal face with the realigned shape exhibiting a tubular figure. After the formation of $\mathrm{C}-\mathrm{C}$ chemical bonds, this carbon then precipitates out with a graphitic structure once the solubility limit within the metal is reached. The size and shape of the catalyst can affect whether graphite, carbon filaments, or carbon nanotubes are formed. According to Deck and Vecchio [7], "as more carbon is deposited on the catalyst it will either diffuse into or over the surface of the particle and become incorporated into the graphitic lattice, increasing the tube's length." Figure 1 shows how, during formation, the catalyst particles will either remain fixed to the substrate (root growth) or detach from the surface and stay encapsulated inside the opposite end (tip growth).

Another mechanism is proposed by Zhang et al. [10] to explain the vapour phase growth of carbon nanotubes. As they describe it, nanotubes nucleate and grow by a tip growth mechanism with metal catalyst particles initially rising with the tube tips as they grow. The particles eventually become fixed in place due to friction between the particles and the tube walls. Deck and Vecchio [7] expand on this theory to explain the growth of nanotubes by tip growth after which friction had immobilised their catalyst and growth had continued slowly open ended.

Chemical Vapor Deposition (CVD) is a popular method for producing CNTs in which a hydrocarbon vapor is thermally decomposed in the presence of a metal catalyst $[8,11]$.
This usually occurs in two main processing configurations, namely, horizontal and vertical. During CVD, a substrate is prepared with a layer of metal catalyst particles, most commonly nickel, cobalt, iron, or a combination [12, 13]. The substrate is then heated to approximately $700^{\circ} \mathrm{C}$ or greater. To initiate the growth of nanotubes, two gases are bled into the reactor: a process gas (such as ammonia, nitrogen, hydrogen, etc.) and a carbon-containing gas (such as acetylene, ethylene, ethanol, methane, etc.). Nanotubes grow at the sites of the metal catalyst, the carbon-containing gas is broken apart at the surface of the catalyst particle, and the carbon is transported to the edges of the particle, where it forms the nanotubes $[11,14]$.

For a successful CVD process, careful choice of catalyst, carbon source, and process/carrier gas must be made. The carrier gas is generally a nonreactive gas that keeps the reactor free from oxygen and provides an inert atmosphere for CNT growth. Argon, hydrogen, and nitrogen are most common but other gases such as ammonia and helium have also been used. Qingwen et al. [15] investigated the effect of carrier gas on the CVD process of cyclohexane and found MWCNTs when argon was used, but when hydrogen was used, some SWCNTs had formed suggesting that careful choice of carrier gas could affect products formed.

\section{Experimental Procedure}

A Swirled Fluid CVD reactor has previously been described elsewhere $[16,17]$ but basically consists of a vertical quartz reactor (1) loaded in a tubular furnace (2) operated between 600 and $1000^{\circ} \mathrm{C}$. A system of valves and rotameters serves to guide flow of carrier gases (in this case argon, Ar) into the vaporizer (3) after which the gas guides the vaporized catalyst and carbon source through a swirled coiled mixer and into the reactor. After the reactor, the carrier gas guides the newly formed carbon nanoparticles (nanoballs, nanofibres, CNTs, 


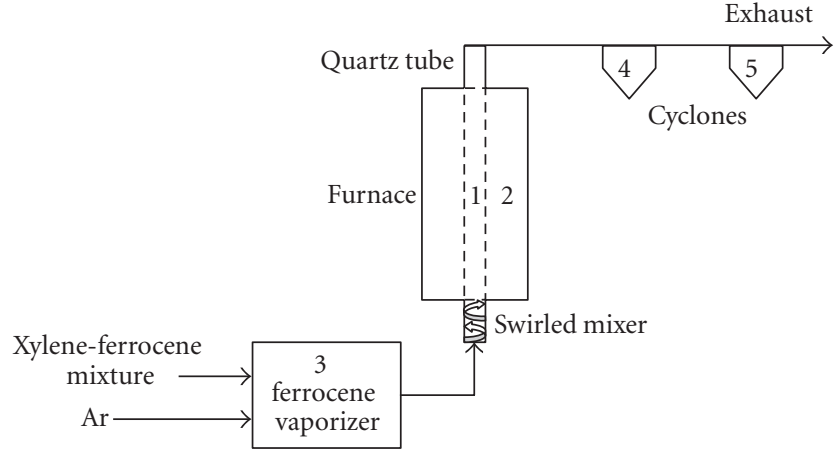

Figure 2: Equipment configuration for Swirled Fluid Chemical Vapour Deposition (SFCVD) reactor.

etc.) as well as any unreacted feeds from the reactor to be collected in the two cyclones $(4,5)$. For the experiments, Xylene will be used as the carbon source, organometallic ferrocene as the catalyst, and a combination of Argon and Hydrogen gas as the carrier gas. Figure 2 presents the proposed equipment set-up for the process.

\section{Results and Discussions}

4.1. Analysis of Experimental Results. The reactor was operated at a temperature of $900^{\circ} \mathrm{C}$ while the feed materials were introduced to the system. Samples were then collected from the hydrocyclone products as well as from material collected from within the reactor. These samples were then analysed by means of a transmission electron (TEM) microscope, the results of which can be seen in Figure 3. Both images shown are of the same scale to illustrate the vast difference in the sample contents. It is immediately evident that the helical and straight nanotubes found in the product are of a much larger diameter than those found within the reactor. This suggests that the mechanism by which the tubes are transported out of the reactor is one of air friction whereby the larger tubes, as they possess a larger surface per weight, provide a larger surface for friction to act, lifting the tubes from the reactor.

\subsection{Effect of Shape and Size on Air Resistance}

4.2.1. Analysis of Forces. Since this work makes use of a vertical reactor with carrier gas passing upward through the reactor column in order to carry the product from the reactor to the hydrocyclones where it can be collected, the primary mechanism by which the product is lifted is one of drag caused by air friction. Figure 4 shows the basic forces acting an object within the reactor.

The weight of the object, $F_{W}$, applies a downward force and is defined as

$$
F_{W}=m g
$$

where $m$ represents the mass of the object and $g$ represents gravitational acceleration, namely, $9.81 \mathrm{~m} / \mathrm{s}^{2}$. The upward moving gas also pushes on the object causing an upward drag force, $F_{D}$, caused by the frictional resistance of the gas passing around the object. For relatively small objects that are roughly spherical in shape, it can be assumed that the drag force is proportional to the velocity giving:

$$
F_{D}=b v,
$$

where $v$ is the velocity of the falling object relative to the flow of gas, measured in units of $\mathrm{m} / \mathrm{s}$, and $b$ is a constant of proportionality called the drag coefficient with units of $\mathrm{kg} / \mathrm{s}$. This type of drag force is called viscous drag. For objects that are not spherical in shape, Newton drag needs to be considered [18] where instead the drag force is described by

$$
F_{D}=\frac{1}{2} C_{D} \rho A v^{2}
$$

where $\rho$ is mass density of the fluid/gas in $\mathrm{kg} / \mathrm{m}^{3}, u$ is the velocity of the object relative to the fluid in $\mathrm{m} / \mathrm{s}, A$ is the reference area in $\mathrm{m}^{2}$, and $C_{D}$ is the dimensionless drag coefficient.

What these three equations show is that in order for any object to be lifted out the top of the reactor, the force acting on the object due to drag has to exceed the force due to gravity. Therefore, by (3), for nanotubes to be easily lifted from the reactor, three main factors can be considered, namely, gas density, gas velocity, and the object's frontal surface area. For these experiments very little can be changed in terms of gas density as the gases were particularly chosen to be an inert carrier gas, Argon, along with Hydrogen to give the best possible opportunity for nanotube production. As for gas velocity, the reactor is already utilizing maximum gas flow and any modification to increase gas flow will most likely result in the feed passing through the reactor with too little time to react and form products. With these two factors remaining reasonably constant, the remaining factors are the drag coefficient and reference area. The larger either of these factors, the more likely the object is to be lifted by the gas out of the reactor.

The drag coefficient, $C_{D}$, is generally not constant for a given body shape. Streamlined objects such as spheres possess low values for $C_{D}$ (well below 1 ) while unstreamlined objects can have coefficients of 1 and greater [19]. In general, the drag coefficient is always associated with a particular surface area [20]. Even when considering different orientations of both helical and cylindrical tubes, a helix is far less streamlined than a cylinder in the same orientation. The loops of the helix provide a larger surface area per tube length resulting in a larger drag coefficient than that of a cylindrical tube.

To properly compare the drag forces, it is also necessary to look at the orthographically projected reference areas of a cylinder and helix, as seen in Figure 5. Since actual elongated tubes in suspension are prone to constantly change orientation as well as being liable to bend, spin, or tumble, it is extremely difficult to calculate the reference areas for all possible circumstances. For this reason, the area comparisons investigated include the orientations where the maximum and minimum reference areas occur, namely, where the tubes are oriented parallel to the direction of gas flow, giving 


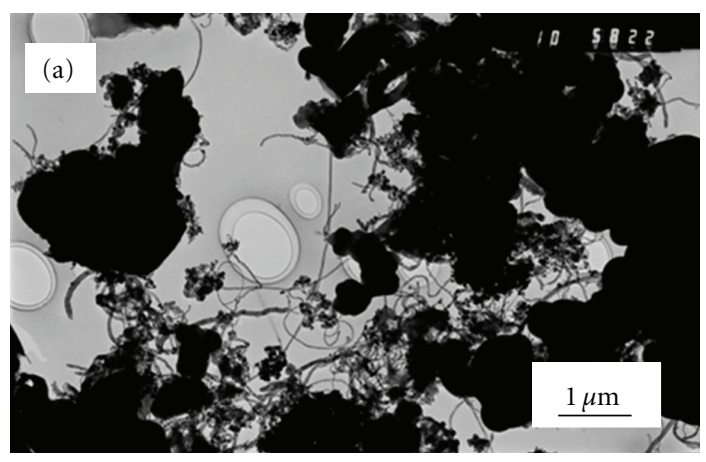

(a)

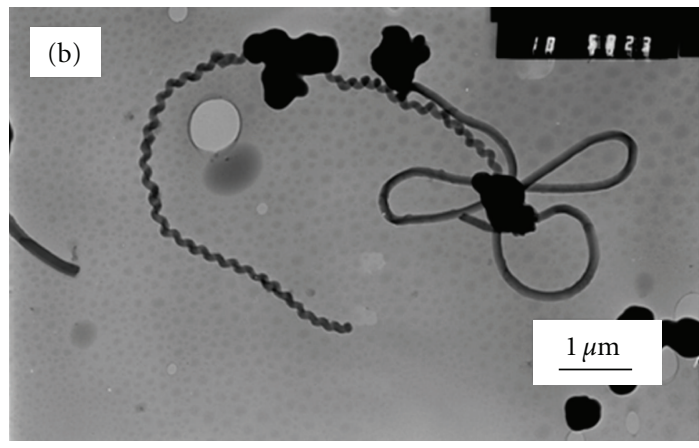

(b)

FIGURE 3: Comparison of images taken from within the reactor (a) and the product (b) at the same magnification for the temperature of $900^{\circ} \mathrm{C}$.

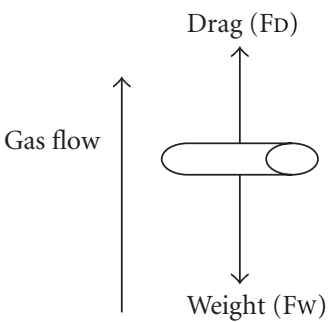

FIGURE 4: Simplified force diagram for an object within the reactor.

the smallest area, and where the tubes are oriented perpendicular to the direction of the gas flow.

4.2.2. Calculation of Reference Areas. Before mathematically comparing the reference areas of the cylindrical and helical tubes, it is necessary to have a clearly defined set of nomenclature:

$A^{\text {par}}$ : Reference area for tubes parallel to direction of gas flow

$A^{\text {perp: }}$ Reference area for tubes perpendicular to direction of gas flow

$r_{t}$ : Tube radius

$r_{h}$ : Helix radius

$L$ : Length of tube

$p$ : Pitch: length of one rotation/coil of the helix

$\theta$ : Angle of rotation in the $x-y$ plane for a helix extending in the $z$-direction.

For a cylindrical tube parallel to the direction of gaseous flow, the orthographically projected area is just a circle of radius $r_{t}$, and thus the area is calculated as

$$
A_{\text {cylinder }}^{\mathrm{par}}=\pi r_{t}^{2} \text {. }
$$

Should the tube be orientated perpendicular to the direction of flow, the resulting reference area is that of an elongated rectangle giving an area of

$$
A_{\text {cylinder }}^{\text {perp }}=2 r_{t} L .
$$

For a helical tube, the reference areas are a bit more complicated. In the instance where the tube is aligned with direction of gas flow, the resulting reference area can take the shape of a circle or of a ring, that is, a circle with a central smaller circle removed. This is dependent on whether the tube radius is smaller than the helix radius or not. This gives for the helix:

$$
\begin{aligned}
A_{\text {helix }}^{\text {par }} & =\pi\left(r_{t}+r_{h}\right)^{2} \quad \text { for } r_{t} \geq r_{h}, \\
A_{\text {helix }}^{\text {par }} & =\pi\left(r_{t}+r_{h}\right)^{2}-\pi\left(r_{h}-r_{t}\right)^{2} \quad \text { for } r_{t}<r_{h} \\
& =4 \pi r_{t} r_{h} .
\end{aligned}
$$

The resulting area for a helical tube perpendicular to flow direction gives a far more complex shape. Changes in both $r_{t}$ and $r_{h}$ can largely affect the given area and as such a more complex mathematical analysis is required. When mathematically analyzing the behaviour of a helix tube, it is necessary to start by observing the behaviour of the helical core. It is known that helices follow certain mathematical parameters, namely:

$$
\begin{gathered}
x=r_{h} \cos \theta, \\
y=r_{h} \sin \theta, \\
z=c \theta
\end{gathered}
$$

for $\theta \in[0,2 \pi]$, where $r_{h}$ is the radius of the helix and $c$ is a constant conversion factor giving the vertical separation of the helix's loops, also equal to $p(2 \pi)^{-1}$. A visual representation of the orientation of the parameters can be seen in Figure 6.

When finding the reference area, it is necessary to find the area between the top and bottom edges of the resulting shape when plotting either $x$ or $y$ against $z$. Figure 7 depicts an example of a possible helix possessing a large tube radius compared to helix radius and a fairly wide distance between peaks.

In order to find the area of a complete tube of variable length, the best approach is to find the area between the top and bottom edges of one coil of the represented helical tube and multiply it by the number of coils in the complete length. The number of coils can be easily found from the tube length 


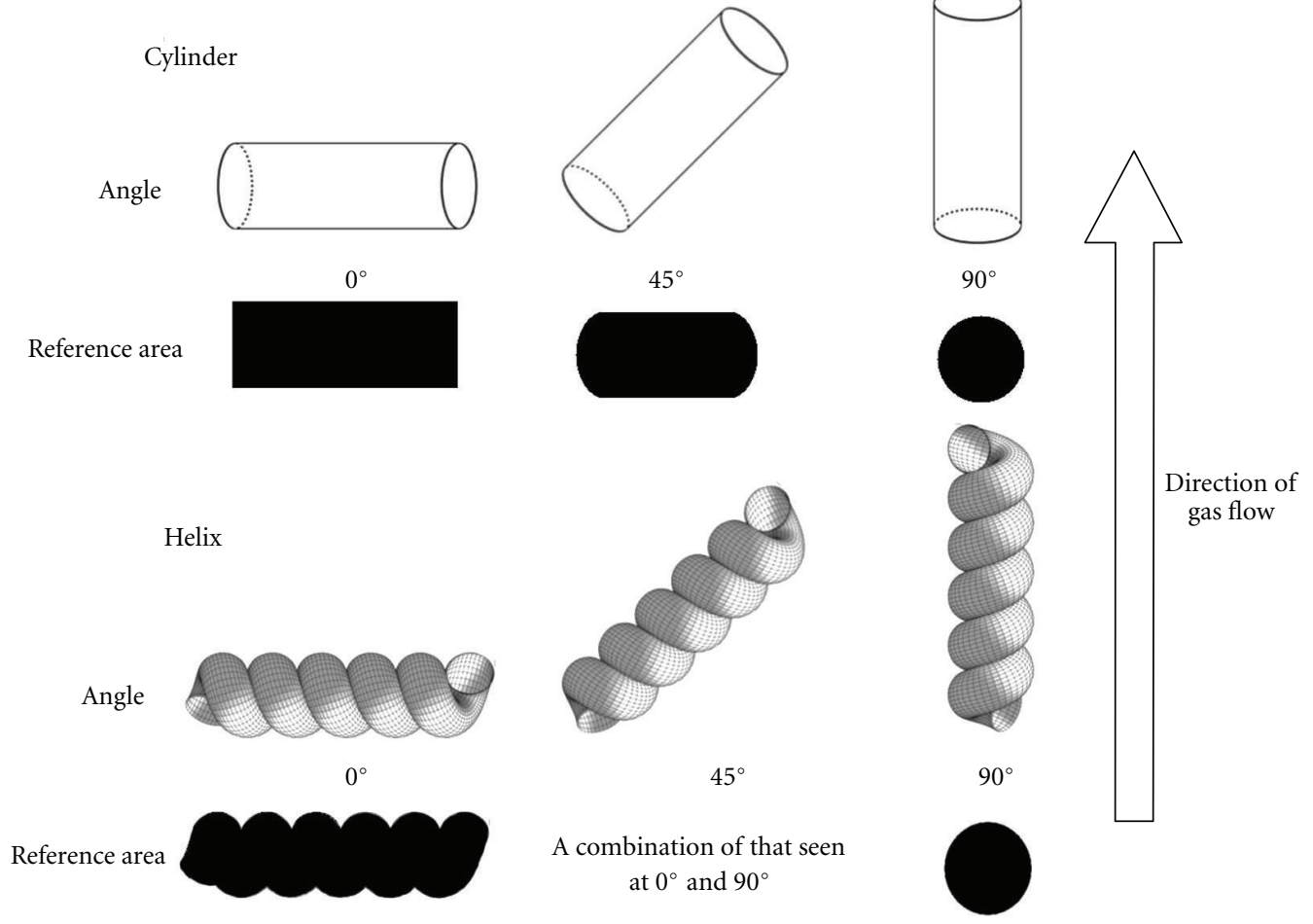

FIGURE 5: A comparison of orthographically projected reference areas for helices and cylinders at different orientations relative to flow of gas.

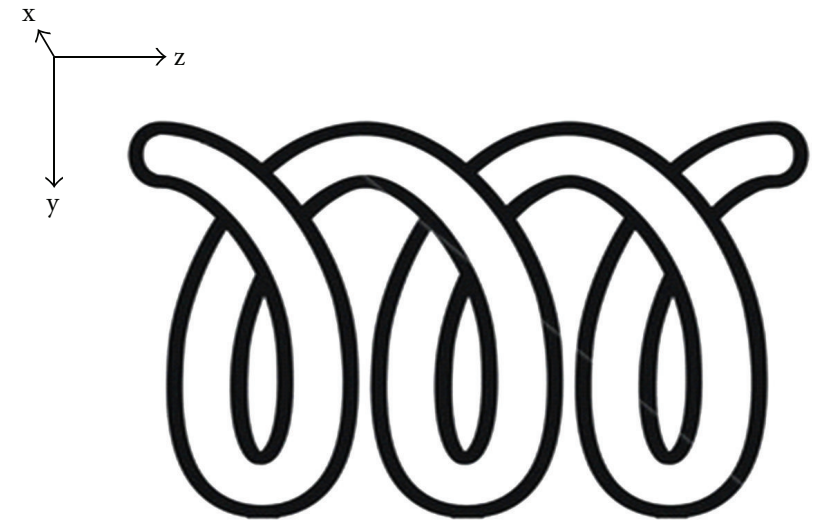

(a)

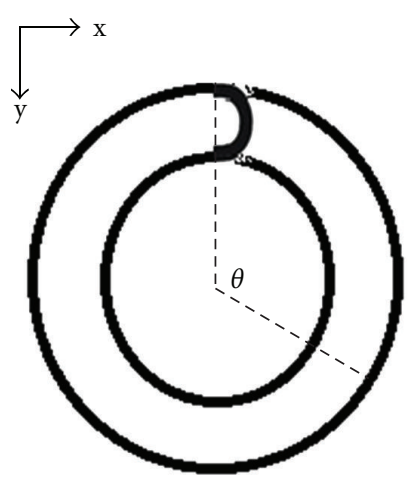

(b)

FIGURE 6: Visual representation of assigned mathematical parameters for calculation.

and length of a single coil. While the core of the represented helix may follow a sine or cosine shape, it is important to remember that the upper and lower edges do not. Being that it is still a tube, the tube itself is one tube radius from the core. Hence, in terms of the represented figure, at all points along the core curve, two points will exist one tube radius away perpendicular to the gradient of the core.
Since it is already known that $y$ is a function of $\theta$ (8), the gradient (in radians), $\psi$, at any point on the core can be found from the following differential:

$$
\psi=f(\theta)=\tan ^{-1} \frac{d y}{d \theta}=\tan ^{-1}\left(\frac{d}{d \theta} r_{h} \sin \theta\right)=\tan ^{-1}\left(r_{h} \cos \theta\right)
$$




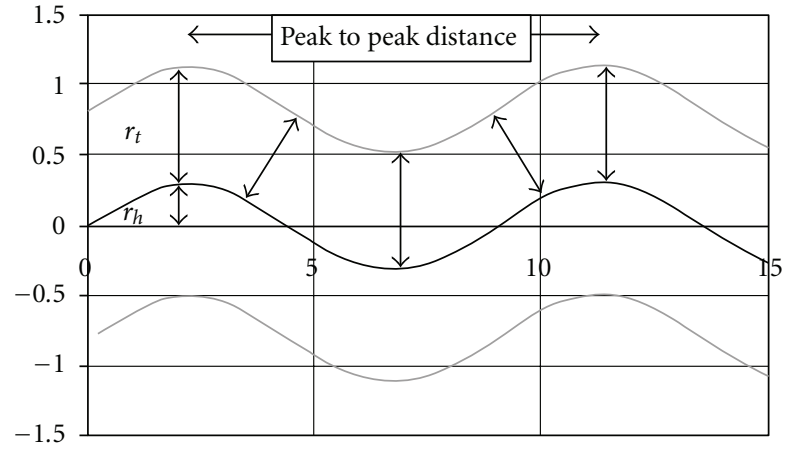

— Helix core

Figure 7: Labelled example of a possible helix.

This gives, for any point along the core, a perpendicular angle, $\psi_{p}$, in radians of

$$
\psi_{p}=\psi+\frac{\pi}{2}=f(\theta)
$$

It is also known that

$$
z=\frac{p \theta}{2 \pi}=f(\theta)
$$

In order to obtain curves for the top and bottom edges, it is possible to plot points one tube radius above and below the core on a plot of $y$ versus $z$ using

$$
\begin{aligned}
& y_{\text {edges }}=y \pm r_{t} \sin \psi_{p} \\
& z_{\text {edges }}=z \pm r_{t} \cos \psi_{p} .
\end{aligned}
$$

This allows the edges to be described as sets of coordinates, the values of which remain dependant on $\theta$ :

$$
\text { Edges }=f(\theta)=\left(z_{\text {edges }}(\theta), y_{\text {edges }}(\theta)\right)
$$

where

$$
\begin{aligned}
& y_{\text {edges }}=r_{h} \sin \theta \pm r_{t} \sin \left[\tan ^{-1}\left(r_{h} \cos \theta\right)+\frac{\pi}{2}\right], \\
& z_{\text {edges }}=r_{h} \sin \theta \pm r_{t} \cos \left[\tan ^{-1}\left(r_{h} \cos \theta\right)+\frac{\pi}{2}\right] .
\end{aligned}
$$

Initially it was hoped that since the equations for the coordinates are both functions of $\theta$, they could be combined into a single equation for the top edge and another single equation for the bottom edge. This would provide a means to determine the reference area of the helix by the difference of the differentials. It was soon determined that while this approach may provide adequate results for simple helix representations such as those seen in Figure 7, it is not suitable for calculating the reference area for more complex helices where the closeness of the loops results in the presence of a visible overlap. A graphical depiction of this can be seen in Figure 8.

While this eliminates the use of equation integration to find the area, graphical methods can still be applied. As the

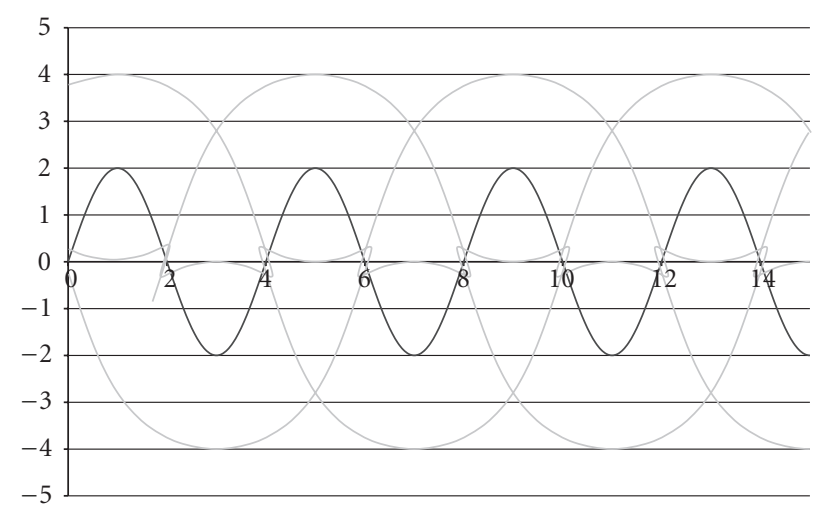

— Helix core

Figure 8: Graphical representation of a complex reference area resulting from visible overlaps of loops.

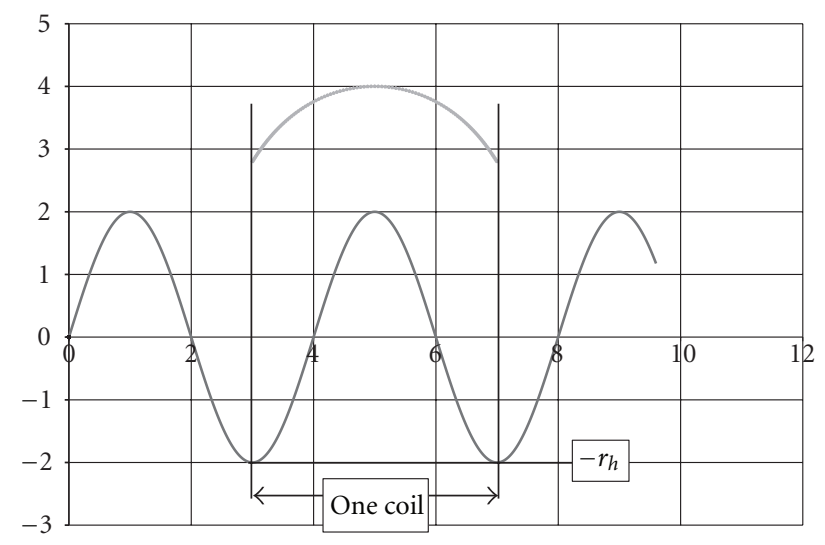

Figure 9: The application of Boolean algebra to leave a single edge for one coil.

graphs are created by plotting new points relative to initial points on the core, by increasing the initial number of points it is possible to increase the accuracy by which the edges are plotted. By applying Boolean logic algebra to the resulting points in Excel, it is possible to exclude the points present in the overlap and leave only those in the uppermost edge. To simplify calculations, the same principles can be used to leave only the points present for one coil, as depicted in Figure 9.

Once the unnecessary points have been eliminated, it is possible to find the area by graphical methods. It is known that the lowest possible point on the graph is negative $r_{h}$. Thus the area can be found from the difference in areas of the top edge and the core to this base. To find the area below the collection of points, summation of a number of vertical bars provided by consecutive points can be performed. In mathematical terms, this is given by, for $k$ points,

$$
\text { Area }=\sum_{i}^{k-1}\left(\frac{Y_{i}+Y_{i+1}}{2}-\left(-r_{h}\right)\right)\left(Z_{i+1}-Z_{i}\right)
$$

where $\left(Y_{i}, Z_{i}\right)$ represents the Cartesian coordinates for each point, $i$, on the graph. 
Once the area below the core has been subtracted from the area beneath the top edge, it can be doubled to provide the reference area per coil. This can be done due to the rotational symmetry of the helix, a consequence of which, for each coil, the area below the core line shall be equal to the area above. While this method may not provide the accuracy that differentiation could, were it possible, it is clear that as the number of defined point's increases, the accuracy of the result nears that of differentiation. As such, by using a high number of points it is possible, with reasonably high accuracy, to calculate the reference area for any given helix.

4.3. Growth Mechanism of Single-Walled CNTs to Helical CNTs. While Section 4.2 shows how the helical shape can contribute to the upward force offered by the extra drag, it does not provide an explanation for the presence of helical nanotubes in the first place. To quote Sharma and Lakkad [21]: "The non-straight, spiral shape twisted filaments or tubes are generated from asymmetrical shaped catalyst particle. Due to this asymmetry of catalyst the carbon atoms traverse across different diffusion path lengths relative to the symmetry axis." What this basically means is that as certain carbon molecules may take a longer time to diffuse into the catalyst particle on one side of the catalyst than the other, the nanotube begins to form faster on one side giving rise to the twisted helical shape. As Li et al. [22] described: "The difference in diffusion through surface and bulk of a catalyst particle results in the difference of precipitation rates among the precipitation facets".

It is believed that multiple possible means whereby the catalyst particle can change shape are possible. One of these methods is a result of catalyst particles deforming due to local poisoning of certain areas of the particle [23]. This results in differences in catalytic activity over the particle with reaction time, consequently resulting in carbon nanotubes with various shapes such as a helix. This implies that the catalyst particles within the reactor can change in shape when exposed to enough heat. As this deformation is random, it is not guaranteed that the resulting shape will give rise to the formation of helical nanotubes which explains the small concentration of helical nanotubes present in the samples. Another possible explanation for the change in catalyst particle shape is the possible presence of weak points on certain catalyst particles. With the flow of gas particles as well as the unreacted material and particles being lifted by the gas, it is a reasonable assumption that the exposed catalyst particle at the end of a growing nanotube could experience an impact from flowing material which could cause the particle to break at any weak points present. It is also possible that while carbon precipitates out of the catalyst particle, any smaller pieces of the catalyst could break off the larger catalyst particle while the nanotube grows. Figure 10 illustrates the change in growth of the nanotube after part of the catalyst particle has broken away. Any of these explanations give an explanation for the existence of straight tubes becoming helical tubes without the observation of a catalyst particle at the junction. It also accounts for the small concentration of helical tubes seen as only certain shapes provide the correct surfaces for helical nanotubes to form.

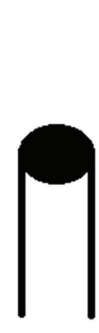

(i)

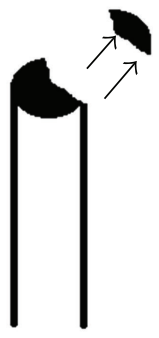

(ii)

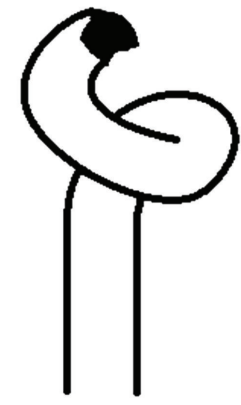

(iii)
FIgure 10: Change in tube growth from cylindrical to helical.

Another aspect to be considered is growth mechanism of the carbon nanotubes within the reactor. If the general assumption of growth is followed, namely, first the metal catalyst adheres to a substrate (in this case the reactor walls) after which carbon diffuses into the catalyst particle and carbon nanotubes begin to form in either a root growth or tip growth manner (as explained in Section 1), then it can also be assumed that the change from straight to helical tubes seen in the results is more likely to occur with tip growth. As the nanotube grows away from the reactor wall, it is exposed to more of the high-temperature gas flowing through the reactor which means that catalyst particles would be more exposed to impact from other particles as well as to more carbon particles with which to promote nanotube growth. This means that under the circumstances that the catalyst particle should deform due to temperature, it will still be provided with carbon atoms from which the helical nanotubes can grow. This also allows for the presumption that if a catalyst particle does change shape and form a helical nanotube, the helical nanotube will be more exposed to gas flow and therefore more likely to be lifted from the reactor.

4.4. Comparison of Helical and Straight Tubes. In order to properly compare the drag forces acting on different tubes within the reactor, it was necessary to investigate the orthographically projected reference areas provided by these tubes. Since the product contains multiple straight and helical tubes of differing length and radius, a comparison of equal length tubes shall be made in order to ascertain the effect the helix shape has on the reference area. For comparative purposes, dimensionless numbers have been chosen arbitrarily, using different ratios of $r_{h}: r_{t}$, to illustrate the different helical shapes' effect on reference area, as well as the effect of the pitch height $p$ (distance between corresponding peaks of the helix coil). A dimensionless length of 100 was used for calculation purposes. Table 1 compares the calculated reference areas for different tube dimension inputs.

What is evident from Table 1 is the fact that, regardless of the structure of the helical tube, the resulting orthographically projected area of a helical tube is always larger than that of a straight tube for both orientations (parallel and perpendicular to the direction of flow), and as such, it can reasonably be assumed that this shall remain the case for 


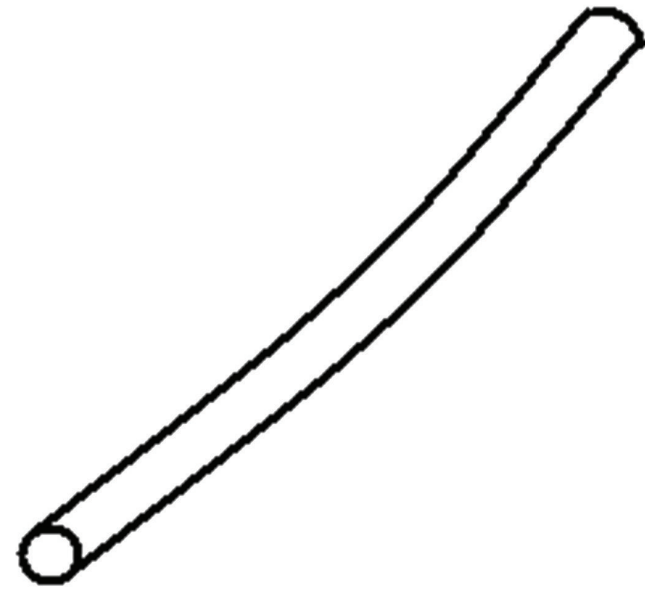

(a)

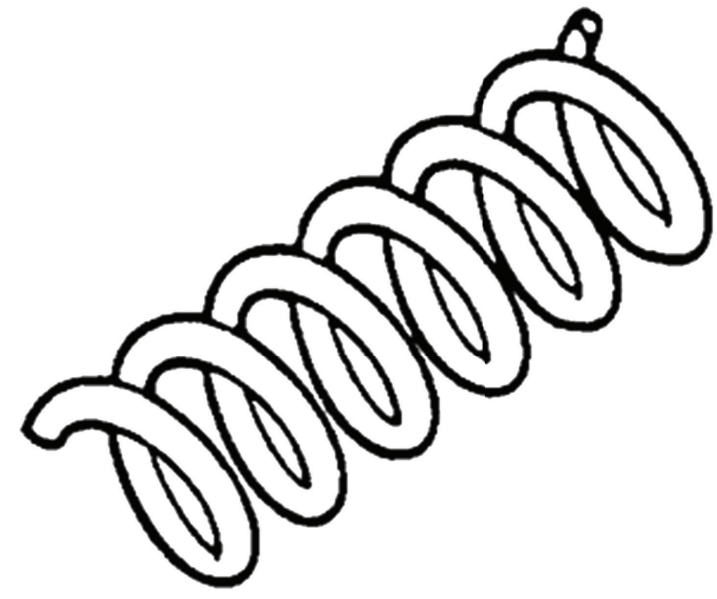

(b)

FIgURE 11: Comparison of a straight tube to a helical tube.

TABle 1: Comparison of calculated reference areas for helical and cylindrical tubes for different tube dimensions.

\begin{tabular}{llcc}
\hline$\left(p, r_{h}, r_{t}\right)$ & Orientation & Cylindrical tube & Helical tube \\
\hline \multirow{2}{*}{$(4,2,1)$} & Parallel & 3.14 & 25.13 \\
& Perpendicular & 200 & 431.63 \\
$(4,1,1)$ & Parallel & 3.14 & 12.57 \\
& Perpendicular & 200 & 284.52 \\
$(4,0.5,1)$ & Parallel & 3.14 & 7.07 \\
& Perpendicular & 200 & 225.08 \\
$(1,1,1)$ & Parallel & 3.14 & 12.57 \\
& Perpendicular & 200 & 379.55 \\
\hline
\end{tabular}

other orientations as well. This coupled with the fact that the more streamlined structure of a straight tube shall also result in a lower drag coefficient, explicitly suggest that the helical shape results in a noticeably higher drag force, and, as such, are far more likely to be lifted from the reactor.

\subsection{Transport Considerations for Helical and Straight Tubes} in the Reactor. The problem that arises with long tubes is that although they possess a large frontal surface area along the face of their length, it is not true for forces acting parallel to their length. This only becomes a problem when it is considered that falling objects tend to move and shift according to the flow of gas. For example, if a tube is slightly curved, the drag force will be larger on the end more perpendicular to the flow causing a larger torque than the end slightly more aligned to the path of flow. What this means is that, while sufficient force to lift the tube may be present while the tube is horizontal, when the tube shifts to a vertical alignment, it may drop more than it was lifted.

The same cannot be said of the helical nanotubes. Although they too possess a far larger frontal area perpendicular to their length than parallel to it, their shape gives far more drag per length at any angle than a straight tube especially when considering gas flow parallel to the length of the tube. With each twist of the helix, a new surface offering resistance is exposed that is not otherwise offering resistance with the straight tube, shown in Figure 11. It is assumed that this explains why almost no purely straight nanotubes are seen in the product for the experiment at $900^{\circ} \mathrm{C}$ with hydrogen. Almost all straight nanotubes seen were attached in some way to a section of helical tubes giving far more drag. This, in turn, provides enough of an upward force to counteract the tubes weight and lift the tubes from the reactor.

Another aspect to be considered is whether or not nanotubes behave the same as larger cylinders as gas flows past them since their size compared to the gas molecules is far smaller than that of a macroscale cylinder to any gas flow. Tang and Advani [24] investigated the drag on a nanotube in uniform liquid argon flow and what they found was that the calculated results for the drag force are different from what one would expect from continuum mechanics. Their work comprised of calculating the drag coefficient from Molecular Dynamics (MD) simulations and comparing it to an empirical equation based on experiments using macroscale cylinders. What was found, surprisingly, was that for a low inlet velocity, the calculated drag from the empirical equation was much smaller than that from MD simulation. At higher flow velocities, the opposite was seen with the simulated drag from MD dropping faster and falling below the empirical result. This was attributed to slippage of the argon atoms on the nanotube at large velocities and confirmed by performing finite element method simulations of flow past a cylinder using continuum approach for the same dimensional similitude. Although this work made use of a liquid Argon flow, it provides a good premise to assume that even with a gas, at higher flow-rates, the drag on a straight carbon nanotubes will be lower than that expected by empirical calculations due to their small size.

Possibly one of the most important things to consider when investigating the effect of the helical shape is that of grouping. Coalescence is far more likely with irregular shapes and helical nanotubes provide far more points 
whereby other tubes can get hooked or stuck than their straight counterparts. Add to this the increased interaction of particles within the reactor due to the swirling action provided by the reactor's design. The resulting clump or cluster of nanotubes would possess a far higher area by which the flowing gas could provide drag by which it would be far more easily lifted from the reactor. This would also help explain the lack of helical nanotubes present within samples taken from reactor walls after the completion of experiments.

\section{Conclusions}

The continuous production of single-walled carbon nanotubes is still a fairly new field of research and a feasible method of a completely continuous production is yet to be proven due to their adhesion to the reactor and pipe walls. During investigations into the use of a swirled-fluid CVD reactor for continuous production, it was found that certain conditions resulted in tubes of varied diameter being found within the reactor itself with the product sample containing trace quantities of helical nanotubes with an approximate diameter below $100 \mathrm{~nm}$, but far larger than some of the tubes that remained in the reactor.

A look into the physics and possible mechanism by which carbon nanotubes are lifted upward out of the reactor column gave some possible rationalization for this. It is theorized that with their near incredibly small weight at such small dimensions, the main lifting force is generated by drag against the frontal surface area of the tube in line with the flow of gas. This also implies that this drag force will be larger for structures with larger frontal surface areas, that is, larger tubes. The helix structure provided by helical nanotubes similarly gives far larger drag per length due to the increased cross-sectional area provided at any orientation with the helix shape. Added to this is the increased agglomeration effect provided by the helical shape. With the far greater lift force acting on helical nanotubes, they are more easily lifted from the reactor explaining the presence of helical nanotubes in the product but not the reactor. While, by comparison, far less research has been performed on investigating the growth mechanism by which helical nanotubes are produced, it is possible that their unique structural properties may provide an improved means by which Single-Walled Carbon Nanotubes can be removed from the Swirled Fluid Chemical Vapour Deposition Reactor.

\section{Acknowledgments}

The authors would just like to acknowledge the assistance of their supervisors, Professor Sunny Iyuke and Professor Frank Kavishe, for their excellent supervision and advice, as well as that of Mr. Georgie Mathews who assisted in the running of certain experiments. The financial assistance of the National Research Fund (NRF) and the African Materials Science and Engineering Network (AMSEN) as well as the assistance of the Centre of Excellence in Strong Materials (CoE-SM) towards the completion of this work is also greatly appreciated.

\section{References}

[1] L. X. Zheng, M. J. O'Connell, S. K. Doorn et al., "Ultralong single-wall carbon nanotubes," Nature Materials, vol. 3, no. 10, pp. 673-676, 2004.

[2] M. S. Dresselhaus, G. Dresselhaus, and P. Avouris, Eds., Carbon Nanotubes: Synthesis, Structure, Properties, and Applications, Springer, Berlin, Germany, 2000.

[3] P. J. F. Harris, Carbon Nanotubes and Related Structures, Cambridge University Press, 1999.

[4] M. Paradise and T. Goswami, "Carbon nanotubesproduction and industrial applications," Materials and Design, vol. 28, no. 5, pp. 1477-1489, 2007.

[5] C. Dekker, "Carbon nanotubes as molecular quantum wires," Physics Today, vol. 52, no. 5, pp. 22-28, 1999.

[6] R. Martel, V. Derycke, C. Lavoie et al., "Ambipolar electrical transport in semiconducting single-wall carbon nanotubes," Physical Review Letters, vol. 87, no. 25, Article ID 256805, 4 pages, 2001.

[7] C. P. Deck and K. Vecchio, "Growth mechanism of vapor phase CVD-grown multi-walled carbon nanotubes," Carbon, vol. 43, no. 12, pp. 2608-2617, 2005.

[8] S. B. Sinnott, R. Andrews, D. Qian et al., "Model of carbon nanotube growth through chemical vapor deposition," Chemical Physics Letters, vol. 315, no. 1-2, pp. 25-30, 1999.

[9] N. Zhao, C. He, Z. Jiang, J. Li, and Y. Li, "Fabrication and growth mechanism of carbon nanotubes by catalytic chemical vapor deposition," Materials Letters, vol. 60, no. 2, pp. 159$163,2006$.

[10] X. Zhang, A. Cao, B. Wei et al., "Rapid growth of well-aligned carbon nanotube arrays," Chemical Physics Letters, vol. 362, no. 3-4, pp. 285-290, 2002.

[11] Y. Ando, X. Zhao, T. Sugai, and M. Kumar, "Growing carbon nanotubes,” Materials Today, vol. 7, no. 9, pp. 22-29, 2004.

[12] N. Inami, M. Ambri Mohamed, E. Shikoh, and A. Fujiwara, "Synthesis-condition dependence of carbon nanotube growth by alcohol catalytic chemical vapor deposition method," Science and Technology of Advanced Materials, vol. 8, no. 4, pp. 292-295, 2007.

[13] N. Ishigami, H. Ago, K. Imamoto, M. Tsuji, K. Iakoubovskii, and N. Minami, "Crystal plane dependent growth of aligned single-walled carbon nanotubes on sapphire," Journal of the American Chemical Society, vol. 130, no. 30, pp. 9918-9924, 2008.

[14] M. Foley, "Carbon Nanotubes: what are CNTs," Carbon Nanotubes, 2006.

[15] L. Qingwen, Y. Hao, Z. Jin, and L. Zhongfan, "Effect of hydrocarbons precursors on the formation of carbon nanotubes in chemical vapor deposition," Carbon, vol. 42, no. 4, pp. 829835, 2004.

[16] S. E. Iyuke, "A Process for Production of Carbon Nanotubes," World Intellectual Property Organization, International Bureau, WO 2007/026213 A1, 2007.

[17] S. E. Iyuke, T. A. Mamvura, K. Liu, M. Meyyappan, and V. K. Varadan, Process synthesis and optimisation for the production of a clean, large quantity of single-walled carbon nanotubes, 2008.

[18] J. Bisquert, P. Ramirez, A. J. Barbero, and S. Mafe, "A classroom demonstration on air drag forces," European Journal of Physics, vol. 12, no. 6, pp. 249-252, 1991.

[19] S. F. Hoerner, Fluid-Dynamic Drag, Hoerner Fluid Dynamics, Brick Town, NJ, USA, 1965.

[20] B. W. McCormick, Aerodynamics, Aeronautics, and Flight Mechanics, John Wiley \& Sons, New York, NY, USA, 1979. 
[21] S. P. Sharma and S. C. Lakkad, "Morphology study of carbon nanospecies grown on carbon fibers by thermal CVD technique," Surface and Coatings Technology, vol. 203, no. 1011, pp. 1329-1335, 2009.

[22] D. W. Li, L. J. Pan, D. P. Liu, and N. S. Yu, "Relationship between geometric structures of catalyst particles and growth of carbon nanocoils," Chemical Vapor Deposition, vol. 16, no. 4-6, pp. 166-169, 2010.

[23] S. Amelinckx, X. B. Zhang, D. Bernaerts, X. F. Zhang, V. Ivanov, and J. B. Nagy, "A formation mechanism for catalytically grown helix-shaped graphite nanotubes," Science, vol. 265, no. 5172, pp. 635-637, 1994.

[24] W. Tang and S. G. Advani, "Drag on a nanotube in uniform liquid argon flow," Journal of Chemical Physics, vol. 125, no. 17, Article ID 174706, 8 pages, 2006. 

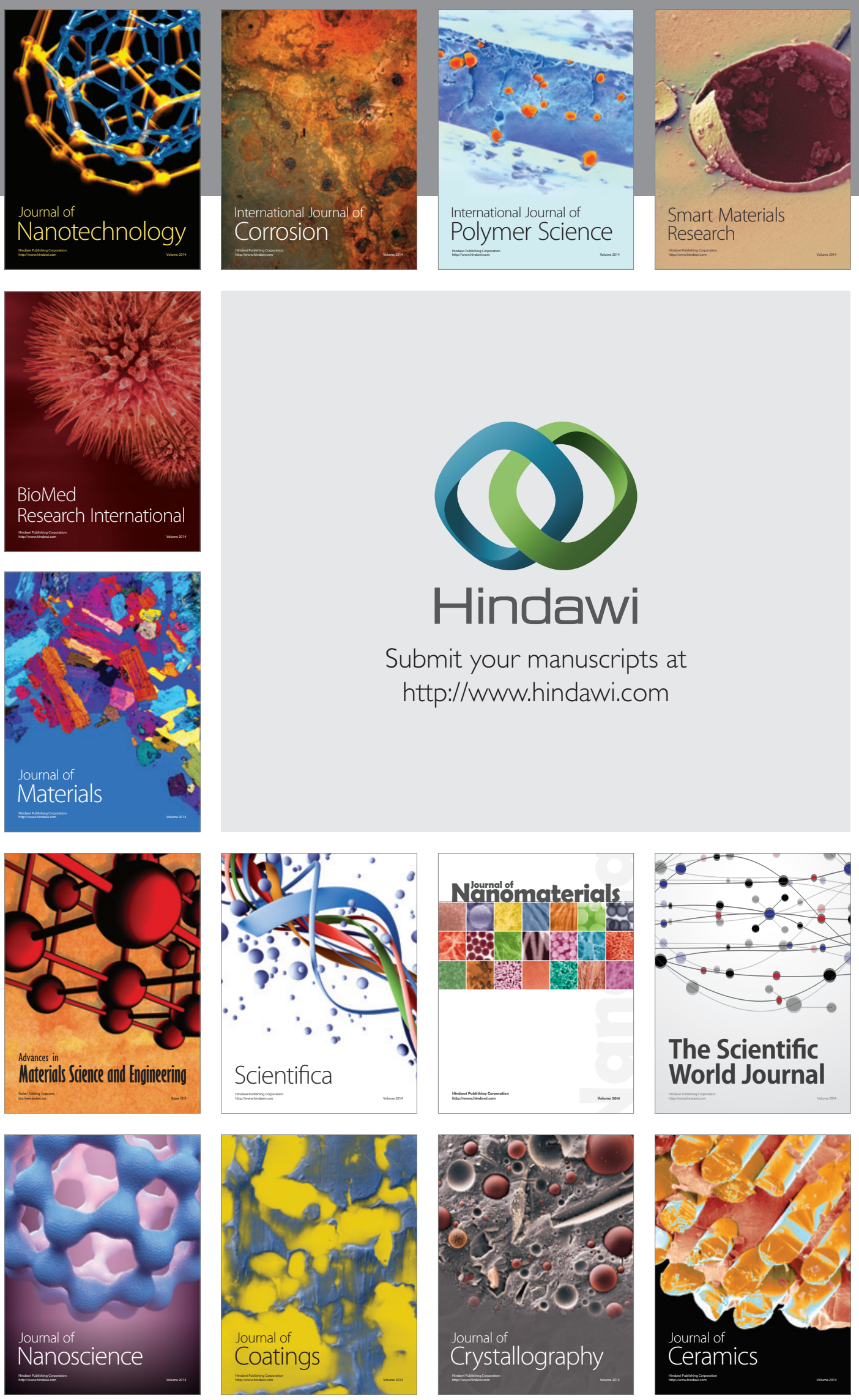

The Scientific World Journal

Submit your manuscripts at

http://www.hindawi.com

\section{World Journal}

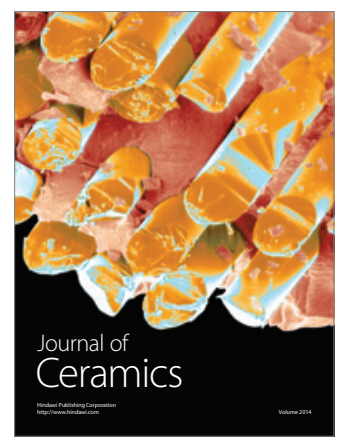

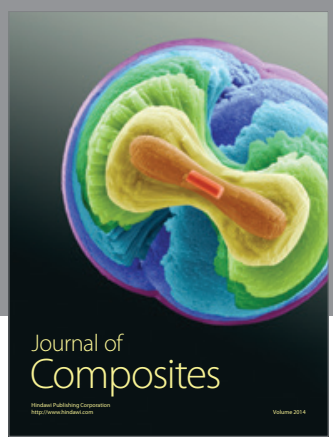
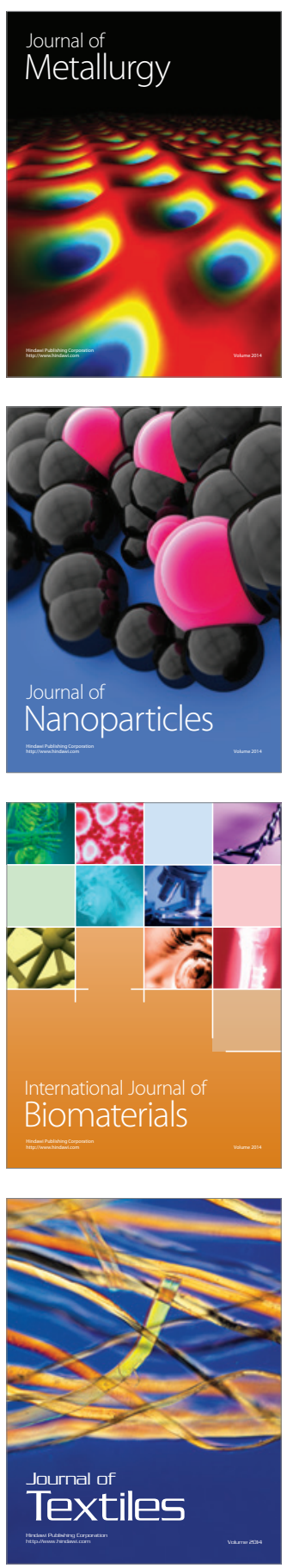